\title{
Norois
}

Environnement, aménagement, société

204 | 2007/3

Patrimoine, culture et construction identitaire dans les territoires ruraux

\section{Les associations culturelles, porteuses de projet pour de nouvelles ruralités?}

Cultural associations, triggering new projects for new country lives?

\section{Estelle Regourd}

\section{CpenEdition}

\section{Journals}

Édition électronique

URL : https://journals.openedition.org/norois/1450

DOI : $10.4000 /$ norois. 1450

ISBN : 978-2-7535-1552-9

ISSN : $1760-8546$

Éditeur

Presses universitaires de Rennes

Édition imprimée

Date de publication : 1 septembre 2007

Pagination : 67-78

ISBN : 978-2-7535-0533-9

ISSN : 0029-182X

Référence électronique

Estelle Regourd, «Les associations culturelles, porteuses de projet pour de nouvelles ruralités? », Norois [En ligne], 204 | 2007/3, mis en ligne le 01 septembre 2009, consulté le 14 janvier 2022. URL http://journals.openedition.org/norois/1450; DOI : https://doi.org/10.4000/norois.1450 


\title{
LES ASSOCIATIONS CULTURELLES,
}

\section{PORTEUSES DE PROJET POUR DE NOUVELLES RURALITÉS?}

\author{
ESTELLE REGOURD \\ Mutations des Territoires en Europe - UMR 5045 CNRS \\ (Université Paul-Valéry), \\ Route de Mende - 34199 MonTPELLIER cedex 5 \\ estelle.regourd@univ-montp3.fr
}

\begin{abstract}
RÉSUMÉ
Dans un contexte de renouvellement des territoires ruraux en France, cet article propose d'analyser le rôle et la place d'associations culturelles et patrimoniales type loi1901 en tant que porteuses de projets dans les zones rurales. Évaluant les qualités de ce type d'acteurs en matière d'implication locale et de construction territoriale, il pointe leurs capacités à décrypter les ruralités émergentes, mais interroge également sur les limites de leurs actions. Les projets définis par ce type de structure sont-ils pérennes et les associations doivent-elles être considérées comme des porteurs de projets efficaces? Le questionnement est mené à partir d'une approche croisée, effectuée en Aveyron et dans les Alpes de Haute-Provence.
\end{abstract}

MotS CLÉS : Action collective - Aveyron - Alpes de Haute-Provence - Associations culturelles et patrimoniales type loi 1901 - Projets territoriaux - Réseau d'acteurs - Ruralité émergente.

\section{ABSTRACT}

\section{Cultural associations, triggering new projects for new country lives?}

This paper deals with the question of the cultural associations as project leaders in rural territories while rural environment is changing in France. Appraising the good points of these collective actors in territorial renovation, it brings out their capacities to decode new ruralities, but it also discusses the limits of their activities. Are the territorial projects defined by these structures sustainable and can the associations be considered as efficient project leaders? This issue is tackled through two areas with different dynamics: Aveyron and Alpes de Haute-Provence.

KEY WORDS : Alpes de Haute-Provence - Aveyron - Collective Action - Cultural Associations - Territorial Projects - Network of players - New Rurality.

En doute-t-on? Les territoires ruraux s'affirment en France, comme lieux de vie, d'activité et de culture. Et de nombreuses zones rurales s'imposent ainsi nouvellement comme cadre de réflexion et de décision à l'échelle locale et globale (Gumuchian, 2000; Perrier-Cornet, 2002). En réalité, elles sont en mesure de le faire car ruraux et citadins les (ré-)investissent activement, que ce soit à 
travers des initiatives individuelles ou des actions collectives. Les projets culturels et patrimoniaux porteurs de ces divers élans sont multiples, mais il est possible d'en décrypter un grand nombre à travers l'instrument associatif. Les structures loi-1901 sont un outil d'action particulièrement accessible et ont des qualités diverses, comme nous le verrons dans une première partie. Leur décryptage permet d'identifier les attentes, les moyens mais aussi les réseaux des acteurs gravitant dans les zones rurales et autour, puis de les analyser. Qu'il s'agisse d'animer, développer une zone via des initiatives originales, construire ou affirmer une identité ou, plus largement, militer pour une idée ou un faisceau d'objectifs imbriqués, la sphère associative s'inscrit souvent au premier rang des porteurs de projets. Ce faisant, elle devient un instrument de décodage des ruralités qui se dessinent, en partie 2. Enfin, une approche croisée de territoires aux dynamiques distinctes en Aveyron et en Haute Provence et porteurs de nombreuses associations relevant des champs culturels et patrimoniaux le met en lumière. Mais, elle permet de s'interroger aussi sur la pérennité des projets territoriaux développés. Des obstacles existent-ils, pouvant freiner les ardeurs associatives locales? De quelles natures sont-ils? Et laissent-ils une chance aux associations de voir aboutir leurs projets?

\section{L'association type loi 1901, outil privilégié du montage de projet}

En France, les associations relevant de la loi 1901 n'ont jamais été aussi nombreuses ni diversifiées. Elles focalisent un nombre d'intérêts croissants des citoyens et de responsables divers. En même temps, elles développent des orientations de plus en plus multiformes, à l'image de la société qui les nourrit. Pour preuve, dans un contexte où les rapports sociaux s'imprègnent, d'individualisme (Crenner, 1997), les Français continuent à intégrer des associations. On compte désormais près de 900000 structures sur le territoire national (Archambault, 2001), réparties dans des secteurs d'activités bigarrés. Sans aucun doute, elles s'affirment donc comme des outils d'action et d'expression prisés des Français, notamment dans les territoires ruraux.

\section{UN INSTRUMENT TERRITORIAL À LA PORTÉE DE TOUS}

Juridiquement parlant, l'association est le cadre par excellence de l'action collective. « Convention par laquelle deux ou plusieurs personnes mettent en commun, d'une façon permanente, leurs connaissances ou leurs activités dans un but autre que de partager des bénéfices » (article 1 de la loi sur la liberté d'association du $1^{\text {er }}$ juillet 1901), elle incarne précisément le moyen de donner corps à un projet collectif. Elle apparaît donc comme un instrument territorial potentiel dont un groupe souhaitant monter un projet peut se saisir dès lors qu'il est constitué. Le même cadre juridique met l'accent sur « la liberté d'association ». Ce droit est fondamental : dans la mesure où les fondements de la structure créée ne remettent pas en question les valeurs morales républicaines, il fait de l'association un instrument accessible à tous. Il est même double puisqu'il juxtapose de façon induite liberté d'association et liberté de l'association, reprenant par-là l'idéal associatif déjà formulé du temps des Lumières. Ainsi, le fait associatif laisse toute sa place à l'expression libre et commune d'un projet pensé par un groupe. En outre, il lui donne une visibilité et une dimension institutionnelle qui lui confère une légitimité et une autonomie, aptes à en faire un instrument prisé des acteurs locaux. Parallèlement, le phénomène associatif est indépendant, du moins devrait-il l'être. Une mainmise sur le système des collectivités territoriales, voire de l'Etat, peut exister, plusieurs auteurs s'y sont intéressés (Cabanes, 1986). Cela dit, elle ne fait pas la règle et n'est qu'un détournement de l'autonomie qui devrait prévaloir. Au fond, l'instrument associatif correspond à « une grande école de responsabilité au service des populations » (Faki, 1997), ce qui fait écho à la notion d'institution, mais la prolonge aussi en renvoyant au concept de démocratie. Cette idée est déterminante : elle institue les associations comme outil élémentaire de la citoyenneté et lui confèrent une mission sociale (Chosson, 1990). 
Sans aucun doute, l'association est donc un instrument institutionnalisé, susceptible de donner aux individus regroupés une emprise forte sur leur territoire à travers un projet collectif. Mais elle présente d'autres intérêts pour les porteurs de projet.

\section{DES QUALITÉS COMPLÉMENTAIRES}

L'une des principales qualités du microcosme associatif tient au caractère à la foi endogène et exogène des associations. Selon G. Di Méo (1991), un acteur endogène est « un acteur [...] s'impliquant de l'intérieur dans une formation socio-spatiale, soit qu'il ait toujours vécu en son sein, soit qu'il vienne d'ailleurs mais adhère à ses valeurs et intègre les normes locales au point d'en imprégner ses représentations et ses comportements ». Il est donc issu de l'environnement socio-spatial dans lequel on l'observe, mais, ce qui est important alors, c'est l'aspect volontaire et réfléchi de cette localisation, l'appropriation et non la localisation en tant que telle. Cela renvoie à l'association, qui, bien que souvent ancrée dans une dimension de proximité pour porter des projets territorialisés (Regourd, 2004), regroupe en son sein des composantes sociales aux origines culturelles et spatiales diverses, cristallisant des représentations parfois divergentes mais toutes unies par un désir d'appropriation de leur environnement. Des sociologues l'ont constaté, la société s'inscrit de plus en plus dans des ancrages multiples, peut-être parce que de plus en plus nomade, plus sûrement parce qu'investie dans des espaces vécus de plus en plus nombreux (Rémy, 1999). En accueillant en son sein des éléments de cette société, l'association fait facilement la jonction entre les dimensions locale et globale et, en cela, est d'autant plus encline à s'impliquer dans les nouveaux territoires ruraux, eux-mêmes ouverts sur des entités spatiales multiples.

Dans un contexte qui fait souvent de la débrouille et du bricolage les bases du développement, faute de représentation suffisante de structures institutionnelles au plan local ou de moyens financiers adéquats, les associations savent aussi faire preuve d'une souplesse exemplaire. On parle d'ailleurs parfois de leur «pouvoir d'ajustement» (Agulhon et Bodiguel, 1981), ce qui en ferait des organes de substitution, sortes de "soupapes de sûreté » (Verrièle et Martin, 1994). Cette caractéristique n'a pas manqué d'attirer l'attention de quelques néo-ruraux soucieux de valoriser leur projet culturel, mais aussi des responsables locaux désireux de trouver des solutions efficaces pour animer durablement leur commune.

Enfin, si pour certains, les associations sont parfois rigides dans leur réglementation juridique ou la définition de leurs objectifs, elles n'en sont pas moins innovantes. M. Bodiguel a reconnu, parmi les premiers, que certaines d'entre elles «sont le résultat d'une ouverture sur la société globale et ses modèles, se faisant non seulement cadre d'absorption de ces nouveaux modèles, mais aussi résistance à la banalisation ». Elles seraient alors un intermédiaire entre la société (rurale ou non) et le nouveau monde rural (Agulhon et Bodiguel, 1981). Mais, l'innovation ne se mesure pas seulement en termes sociaux et peut avoir une portée globale, intéressant l'ensemble des composantes d'un village... Dans ce cas, les associations proposent une assistance d'ordre social pour se repérer face aux changements, mais elles initient elles-mêmes des mutations notamment en matière de culture, devenant « de véritables organisateurs villageois, mais surtout d'excellents laboratoires [...] d'idées » (Fougerouse, 1996). Au-delà, les associations évoquent les notions de diagnostic et d'accueil et démontrent leur capacité à effectuer une évaluation du potentiel existant sur le plan patrimonial et culturel. Plus précisément, elles sont capables de «formuler les préoccupations locales » (Bachelard, 1993) et de « défricher des demandes sociales émergentes » (Nyssens, 1998). Cette notion de diagnostic, interchangeable avec celle de travail d'amont, est importante : elle fait des associations un outil d'approche d'un espace précis et s'avère indispensable à tout projet de développement local. Cela renvoie au fondement même de ce qu'est une association, un groupe d'accueil par excellence, mais cela fait également du secteur associatif un outil d'ouverture d'un espace vers l'extérieur. 


\section{UNE DYNAMIQUE GLOBALE QUI S'AFFIRME AUSSI EN ZONE RURALE}

Nul doute que les associations ont trouvé leur place comme instrument de valorisation de projets. Leur succès en France permet de l'affirmer. Et bien qu'aucun recensement global basé sur la différenciation urbain-rural n'ait encore été fait à ce jour, l'observation de la répartition géographique de la dynamique associative ${ }^{1}$ révèle indirectement un poids comparable des associations dans les zones urbaines et rurales, récusant ainsi l'idée reçue de la suprématie des villes en la matière. Selon une analyse au niveau départemental (figure 1 et tableau 1), les territoires à dominante rurale se prévalent en effet de taux de créations aussi forts, voire supérieurs à certains taux observés dans les territoires à dominante urbaine. Ainsi, parmi les vingt départements aux taux de création les plus forts en 2000 et si l'on se réfère parallèlement au Zonage en Aires Urbaines (INSEE, RGP 1990), douze, soit plus de la moitié, peuvent être considérés comme des zones territoriales à dominante rurale : la Lozère, la Corse, l'Ariège, les Pyrénées Orientales, les Alpes-de-Haute-Provence, les Hautes-Alpes, l'Aude, le Lot, la Savoie, le Gers, le Tarn-et-Garonne et l'Aveyron. Une échelle d'analyse plus fine serait précieuse. Pourtant, les observations faites à partir de la maille départementale confirme déjà les propos de M.-F. Épagneul et N. Mathieu (2000), pour qui, comme l'ensemble des associations, les associations rurales se sont multipliées et développées depuis les années 1980 et sont aujourd'hui particulièrement nombreuses. C'est d'autant plus vraisemblable qu'en tant qu'espace de faible densité, donc en tant qu'espace qui a des besoins de sociabilité marqués, l'espace rural a pu s'affirmer et s'affirme encore comme un des lieux particulièrement favorables au développement de projets divers et innovants.

\section{Des projets territoriaux multiples pour des attentes socio-spatiales diversifiées}

Grâce à son hétérogénéité, le monde associatif est capable de porter des projets extrêmement variés. Si un grand nombre de ces derniers ont une vocation territoriale en zone rurale, entendez qu'ils apparaissent souvent comme des supports du développement territorial (Regourd, 2004), ils prennent des formes particulièrement diverses. Une approche croisée de sphères associatives rurales aveyronnaise et provençale ${ }^{2}$ l'a mis en lumière, donnant un aperçu des ruralités qui se dessinent.

\section{Pour une REVITALISATION LOCALE}

Parfois justement décrites comme moteurs de territoires en mouvement (Favey et Pellerin, 1999), certaines associations ont un pouvoir réel dès lors qu'il s'agit de dynamiser, animer, stimuler leur environnement local. Elles sont nombreuses à vouloir faire de leurs territoires d'attache des territoires vivants. Mais ce pouvoir n'est pas uniforme, ni même toujours aussi efficace. Il se décline, en fait, selon au moins trois champs.

Les structures rurales ont souvent un rôle majeur sinon essentiel en matière de support à l'installation. On pense à celles qui fournissent des services, tels la garde d'enfants, mais il en est d'autres à vocation culturelle qui comptent également. Ces dernières garantissent une animation régulière au plan local. Le plus souvent, elles proposent des rencontres de qualité dans des communes éloignées des centres urbains, alors que ces derniers sont encore trop fréquemment considérés à tort comme seuls pourvoyeurs de culture. En plus d'évènements alléchants, elles offrent alors des lieux de sociabilité et de convivialité. Or, c'est un élément potentiellement déterminant pour

1. Disponible sous forme de tableaux et de cartes, dressées à partir des déclarations au Journal Officiel depuis 1975, dans l'ouvrage du CNVA, 2003, p. 92 et suivantes.

2. Travail de terrain et entretiens menés auprès de responsables associatifs, dans le cadre d'un doctorat de géographie sur le phénomène associatif dans la recomposition territoriale rurale, soutenu le 02 octobre 2004 à Montpellier, sous la direction d'A. Berger et M.-C. Maurel. 


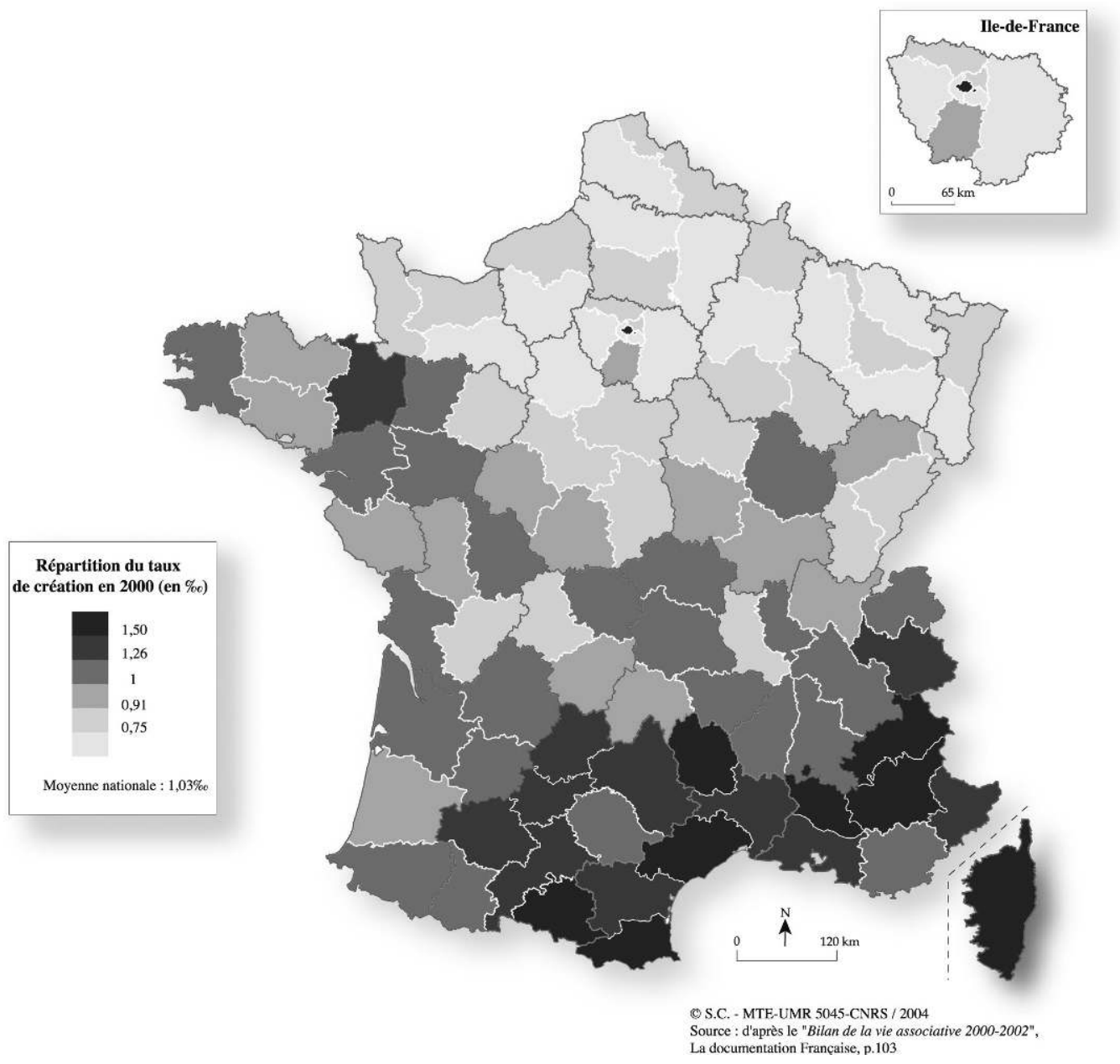

Figure 1 : Le taux de création des associations pour 1000 habitants en 2000, par département en France Rate of association creation, per 1000 inhabitants, in France, by departements in 2000

des néo-ruraux sans attache au plan local. À leur manière, ces associations contribuent à asseoir l'attractivité du lieu. Et qu'elles soient fréquentées ponctuellement, ou hissées au rang de véritables animateurs de la vie rurale, elles sont unanimement reconnues comme indispensables au développement territorial. C'est que l'on a pu observer en Luberon (Alpes-de-Haute-Provence) où une association de cinéma itinérant installe sa toile régulièrement. Elle a vu le jour en 1996, à Pierrevert, pour « développer le cinéma à la campagne pour tous ». Aujourd'hui, Cinéma de Pays, a trouvé son public. Et sans aucun doute, la structure participe à sa manière à faire du Luberon rural un territoire vivant et attrayant.

Les associations rurales mobilisent parfois des salariés pour faire vivre leur projet ou même fonctionner, créant ou induisant une activité. Ce type de questions a été traité de façon précise à travers l'angle économique et rural (Épagneul, Mathieu, 2000). Pourtant, un point demeure peu étudié : une association peut rendre l'économie locale pérenne les douze mois durant. Cette idée se défend sur de nombreuses communes et illustre la volonté de groupes locaux de valoriser leur cadre de vie, tout en le défendant contre une sclérose éventuelle induite, par exemple, par un 


\begin{tabular}{|l|c|l|c|}
\hline \multicolumn{1}{|c|}{ Départements } & $\begin{array}{c}\text { Taux pour 1 000 habitants } \\
\text { (en 2000) }\end{array}$ & Départements & $\begin{array}{c}\text { Taux pour 1 000 habitants } \\
\text { (en 2000) }\end{array}$ \\
\hline 1. Paris & 2,10 & 11. Aude* & 1,41 \\
2. Hérault & 1,91 & 12. Bouches-du-Rhône & 1,41 \\
3. Lozère* & 1,81 & 13. Lot* & 1,37 \\
4. Corse* & 1,71 & 14. Savoie & 1,33 \\
5. Ariège* & 1,71 & 15. Gers* & 1,33 \\
6. Pyrénées-Orientales* & 1,65 & 16. Haute-Garonne & 1,30 \\
7. Alpes-de-Hte-Provence* & 1,63 & 17. Tarn-et-Garonne* & 1,30 \\
8. Hautes-Alpes* & 1,54 & 18. Aveyron* & 1,27 \\
9. Vaucluse & 1,51 & 19. Ille-et-Vilaine & 1,26 \\
10. Gard & 1,48 & 20. Alpes-Maritimes & 1,26 \\
\hline
\end{tabular}

* départements ruraux

Source : E. Regourd, 2004, d'après C.N.V.A., 2003

Tableau 1 : Classement par ordre décroissant des créations d'associations pour 1000 habitants par département Down ranking of association creations, per 1000 inhabitants, in France, by departements in 2000

tourisme saisonnier ou une agriculture en difficulté. Les Amis de la Couvertoirade, dans le SudAveyron (Aveyron), mènent en partie cette bataille. Créée en 1968, dans une cité templière très touristique, cette association à vocation patrimoniale a progressivement amorcée une réflexion sur la « qualité de la vie », ce qui lui a permis, selon son président, d’interpeller les adhérents sur la question « des moyens à se donner vis à vis de la délicate question des apports saisonniers du tourisme ». Aujourd'hui, la commune compte 153 habitants l'hiver (INSEE, RGP, 1999) et accueille «plus de 100000 visiteurs l'été ». Or, plutôt que de rejeter le tourisme en bloc, tout en ayant abandonné l'idée d'imposer quoique ce soit, faute de moyens, l'association organise des séances publiques de concertation et, "grâce à une action de valorisation du village ", est parvenue " à attirer des artisans permanents ». Les associations comme défenseurs actifs du lieu se font donc bien parfois les garantes de la vitalité territoriale. Du moins, en sont-elles les principales forces de réflexion.

Sur le plan social, l'apport des associations se situe couramment au niveau de la vie quotidienne. Les associations d'aide à domicile, ADMR, qui maintiennent une vie localement grâce à l'accompagnement des personnes âgées, illustrent bien cela. Mais, un autre type d'initiatives, faits d'associations socio-culturelles, est tout aussi intéressant. Certaines structures d'animation s'attachent à faciliter et enrichir la vie des gens par la formation, l'accès à la culture, la convivialité... Associations généralistes a priori, elles sont en fait axées sur la vie et la localité. La Cardabela, à Nant (Aveyron), s’inscrit dans cet esprit. Créée en 1987, sous l'égide de l'union «Peuple et Culture ", elle affiche clairement la volonté de "sauvegarder un tissu associatif et rural vivant » et développe des projets qui «puissent répondre aux attentes et aux besoins réels des habitants ». S'éloignant de « l'intellectualisme des débuts » insufflé par le réseau national, selon son président, elle s'intéresse alors davantage à « la vie de proximité ». Un atelier d'initiation à l'informatique, plébiscité par les habitants, a ainsi été récemment mis sur pied. Or, pour beaucoup, il constitue un excellent support d'ouverture à la culture : via l'accès à Internet, il permet aux utilisateurs de surfer sur des sites liés à l'art, l'environnement, l'éducation, les loisirs..., de choisir et de commander des livres en ligne, de s'abonner aux principaux journaux nationaux, mais aussi de se tenir informés des divers évènements culturels locaux ou extra- locaux, grâce à des sites tels que celui de la Maison du Livre ${ }^{3}$, librairie ruthénoise particulièrement dynamique sur le plan culturel, celui du réseau « Peuple et Culture ${ }^{4}$ », ceux des divers réseaux associatifs partenaires de la Cardabela, 
etc. Les associations ont fort à faire en matière d'amélioration des conditions de vie en milieu rural. Certaines s'y emploient, avec enthousiasme et créativité. Le caractère original et innovant de leurs actions est d'ailleurs souvent leur seule arme pour valoriser leur projet.

Ces trois exemples donnent un aperçu de ce que des organisations associatives sont capables de faire pour garantir un minimum de vitalité à leur territoire. Loin de n'être que des cas particuliers, ils sont très représentatifs de ce qui se projette dans les campagnes. Plusieurs zones visitées ont montré en effet qu'elles disposaient d'au moins une de ces structures.

\section{DE LA CULTURE À LA DÉFENSE DE LA NOUVELLE RURALITÉ}

Cependant, les associations savent dépasser le cadre de la proximité pour développer un projet plus global. À travers leurs universités rurales, colloques, séminaires et conférences..., elles sont sans aucun doute des moteurs essentiels de la réflexion sur l'évolution du monde rural français. On partage donc le point de vue de F. Sanselme (2001), pour qui « il existe aussi, du côté des acteurs sociaux ordinaires, une théorie structurée et rationnelle de la ruralité qui tente de comprendre et d'ordonner le monde». Tout comme lui prend en compte les Maisons Familiales Rurales, on considère les associations comme « parties prenantes intégrantes d'une coproduction conceptuelle de la ruralité ». Simplement, leur participation en ce sens n'est pas toujours formelle.

En réalité, quelques structures 1901 se font partisanes d'une convergence territoriale au-delà du rural. Dans ce cas, leur discours n'est ni ruraliste, ni ruralisant. Simplement, elles font de l'urbanité et de la ruralité deux versants d'un même monde étroitement imbriqué qui leur donne, au fond, le rôle d'« indifférenciateur territorial ». Parmi elles, se trouve l'Association d'Histoire et d'Archéologie du Grand Luberon, à vocation patrimoniale. Cette dernière se défend de vouloir « uniformiser » l'offre culturelle, par rapport à un référent qui serait issu du monde urbain. Simplement, elle est attachée à son caractère « a-spatial ». Ainsi, selon sa présidente la structure s'est créée sur la commune de Céreste "parce que cette dernière portait des richesses archéologiques », mais, " cela aurait pu tout aussi bien être dans les faubourgs d'Apt». La localisation dépend de l'existence de champs de fouille et ces derniers ne sont par définition ni ruraux ni urbains mais « situés n'importe où ». Ce qui est important, c'est que l'association «diffuse [ensuite] les découvertes faites et les connaissances acquises, auprès des habitants du pays comme auprès des touristes, quelle que soit leur provenance ». Pas de débat rural-urbain, donc. Simplement, la volonté de «faire converger» des initiatives et la curiosité des gens pour l'histoire, qu'ils soient de la ville ou de la campagne, d'origine citadine ou rurale...

Pourtant, face à cela, de nombreuses associations revendiquent ouvertement une participation globale à l'élaboration de la « ruralité ». Cette dernière apparaît alors la plupart du temps comme un atout à mettre en avant dans un souci de développement. Grâce à la préexistence d'une équipe de pilotage d'un Programme Pour la Revitalisation du Monde Rural, impulsé par la Mutualité Sociale Agricole et dont les maires locaux ont repris l'initiative, l'association PARM, de SaintAmans-des-Côts (Aveyron), aujourd'hui dissoute, avait ainsi pour but explicite « de contribuer à la restauration du tissu social rural et au développement d'un environnement accueillant », en s'appuyant notamment sur « la réhabilitation du patrimoine bâti de caractère ». Bien que construit autour d'actions précises, le projet était donc bien global et défini autour de la question du devenir rural, tandis que l'association faisait de la ruralité la sève du développement local.

Mais le but peut-être différent : redonner au monde des campagnes une visibilité et, par extension, une identité occultées par la prédominance des villes. Dans ce cas, les associations servent de laboratoire à l'élaboration d'une ruralité sur mesure, parfois artificielle, parfois inspirée d'un modèle séculaire ou parfois construite à la croisée de représentations diverses. Dans tous les cas, il s'agit bien « d'une ruralité qui constitue une pratique évolutive à identifier et interpréter et non une donnée naturelle, circonscrite a priori - tout espace pouvant être l'objet de formes variées d’appropriation symbolique ou matérielle » (Sensébé, 2002). L'association TLP (Télévision Locale de Pays), basée à Céreste en Luberon (Alpes-de-Haute-Provence) et qui revendique une vocation 
culturelle dans la mesure où elle « collabore avec les principaux organismes culturels locaux », « offre une formation à l'audiovisuel » et « diffuse des programmes de qualité », cristallise parfaitement cet enjeu. Selon son président, l’instauration d'un « réseau de télévision local » permet de «créer un outil d'information de proximité » et donne l'occasion à « une zone très rurale » de « créer elle-même son support d'information, avec les technologies les plus avancées ». Dans le même temps, elle conforte l'identité locale, faisant écho aux propos de S. Clairet (2000) pour qui « il apparaît nettement que la télévision régionale [...] participe d'un processus de réappropriation de l'espace régional ». Par l'un des médias les plus répandus actuellement, il s'agit donc de donner à voir la ruralité telle qu'elle est désormais.

D'autres associations, enfin, mettent en avant des préoccupations plus restreintes a priori, comme l'aménagement du territoire ou la démocratie locale participative..., mais qui sont autant de thèmes transversaux susceptibles d'illustrer ce qu'est la ruralité d'aujourd'hui. Le cas de Patrimoine de Vachères (Alpes-de-Haute-Provence) éclaire, par exemple, le jeu associatif dans les discussions préparatoires à la mise en place de territoires de projet. Concernée par le « Pays de Haute-Provence ${ }^{5}$ », cette structure « citoyenne volontaire » s'est engagée, selon son président, à « provoquer le débat » localement, au cours de simples « réunions publiques à la salle polyvalente ». Elle "se charge alors du travail d'épluchage et de compréhension » autour des questions que soulève notamment l'intercommunalité, pour le « retransmettre » ensuite auprès des gens qui le souhaitent, villageois ou élus. Au-delà d'une mobilisation unique pour préserver le patrimoine rural, elle participe donc à un débat plus large, concernant le devenir institutionnel de son cadre de vie. Bien évidemment, toutes les associations ne s'engagent pas comme cela. Mais cet exemple en dit long quant au potentiel associatif en matière de réflexion sur des thèmes aussi complexe que l'intercommunalité ou la constitution des Pays et la place qu'il faut accorder à la ruralité dans leur définition. Ces questions rejoignent celle de la démocratie locale, car si les associations se positionnent ainsi, c'est que leurs adhérents souhaitent s'exprimer sur la gestion, l'organisation et la valorisation de leur cadre de vie. Et même si ces débats sont parfois informels, générés dans le feu de l'action, qu'importe! En tant que structure collective, leurs membres, leurs adhérents... se chargeront d'essaimer les idées, les échanges et les propositions, quitte à les reprendre ensuite sous forme de débats plus cadrés.

\section{Du projet territorial à la mise en acte : quelle réalité?}

Entre le moment où les associations formulent le projet qu'elles souhaitent valoriser et la mise en acte de ce projet, il existe parfois un décalage tel qu'il laisse à penser que le projet ne sera jamais monté. Plus souvent qu'elles ne le croient, les structures se heurtent en effet à des obstacles bien réels, qui, s’ils ne sont pas toujours infranchissables, sont aussi divers que variés.

\section{LES RÉSEAUX D’ACTEURS, UN OUTIL À DOUBLE TRANCHANT}

L'insertion dans le jeu des acteurs territoriaux est un levier essentiel pour accéder à l'action territoriale. Si elle n'est pas nécessaire en soi, elle permet d'acquérir un certain nombre de soutiens, bienvenus dès lors qu'on incarne une structure isolée, porteuse de projet. Mais cette implication ne se fait pas toujours sans heurts.

Les associations sont un cadre privilégié d'émergence de conflits. On veut parler de conflits d'usages entre des groupes distincts, mais aussi d'autres types de clivages, opposant les associations aux acteurs territoriaux. Il est primordial de percevoir ces lignes de tension dans la mesure où leur développement peut provoquer le blocage d'un projet commun, en empêchant la convergence des forces en présence. 
La confrontation oppose souvent une association aux acteurs locaux les plus proches d'elle, tels qu'une mairie et tandis que l'un tente d'instrumentaliser l'autre, le deuxième tente, soit, de se défendre, soit, plus ambitieusement, de se hisser en contre-pouvoir. À Reillanne (Alpes de Haute-Provence), les tensions de ce type semblent mineures. Les maires ont-ils su susciter un assentiment général? Rien n'est moins sûr, mais les municipalités étant bien souvent les premières sources de subventions, les petites structures hésitent avant de fronder. Durant les entretiens, des associations dites « de services » ont pourtant dénoncé, parfois à demi-mot, ce qu'elles considèrent comme des « manœuvres politiciennes ». L'École de Musique et l'Office de Tourisme se sont ainsi insurgés contre le positionnement de municipalités du canton qui feraient impunément profiter leurs administrés des prestations offertes, sans jouer le jeu des subventions par ailleurs. Chez les responsables associatifs, née le sentiment d'être utilisé en même temps que d'être paralysé, créant les premiers rapports de force entre des acteurs potentiellement alliés sur des projets territoriaux communs.

D'autres situations opposent parfois des associations à des acteurs de la sphère économique ou pour des raisons économiques. Dans ce cas, les différents ne soulèvent plus des enjeux de pouvoir, mais relèvent davantage de la concurrence ou de l'empiétement sur les domaines respectifs. L'association Cinéma de Pays (Alpes-de-Haute-Provence), évoquée par ailleurs, a glissé quelques mots à ce sujet. Son différent l'oppose aux salles de cinéma « classiques » de Manosque et Digne, qui craignent pour leur clientèle. Une entente pourrait certainement être trouvée, mais alors que, depuis peu, l'association propose, en plus de ses tournées classiques " un été au cinéma », événement annuel à Manosque, les rapports se tendent et tournent à la « concurrence ouverte ». Fantasme ou réalité, les acteurs s'écartèlent, au lieu d'être dans la concertation et la complémentarité sur un même territoire.

Les conflits de personnes, plus difficiles à appréhender, surgissent enfin invariablement. Généralement, ils reposent sur des différences de perceptions et de vécus du territoire, opposant des acteurs d'origines socio-spatiales et de milieux différents. À Reillanne, l'association culturelle Témoignage Contemporain suscite quelques émois. Le différent a été évoqué du bout des lèvres, mais diverses discussions avec des personnes extérieures à l'association ont montré qu'une partie des villageois se sentait « loin des préoccupations » des artistes menant l'association. Bien malgré elle, l'association réveille des tensions sociales et culturelles, qui pourraient bien avoir un impact réel sur la construction territoriale en cours. Ce n'est probablement pas sans lien avec les questions d'appartenance et d'identité, piliers forts d'un territoire équilibré. Les membres de l'association sont pour beaucoup des nouveaux résidents aisés, installés depuis quelques années. Et c'est aussi à travers l'implication active dans leur projet culturel qu'ils ont forgé leur appartenance au lieu. Mais en se mobilisant ainsi, ils ont parfois oublié de faire le lien avec l'ensemble des résidents. Dans le même temps, les deux groupes ne portent pas les mêmes idéaux concernant l'identité territoriale. Tandis que les derniers arrivés voudraient lui attacher une dimension " paysagère et artistique » forte, les autres revendiquent avant tout la dimension « du travail et de la solidarité ». Des attentes en termes de valorisation territoriale s'affrontent sans aucun doute.

La négociation autour d'un projet se grippe donc occasionnellement, réduisant les chances d'une concertation collective entre les acteurs territoriaux. Des associations se retrouvent isolées ou se rétractent par manque de recul, prudence ou même méfiance. Dans tous les cas, elles se mettent en retrait d'une partie du jeu territorial local, amoindrissant la possibilité de mener à bien leur propre projet.

\section{MENER UN PROJET À TERME, UNE DIFFICULTÉ POTENTIELLE}

Au-delà des obstacles rencontrés lors de leur insertion dans un jeu d'acteurs, les associations sont parfois à l'origine de l'échec du développement de leur projet, soit qu'elles se dispersent en chemin, soit qu'on leur mette des bâtons dans les roues. 
L'action d'une association peut avoir ainsi des effets ambivalents pour le territoire. Ses activités peuvent conforter les dynamiques territoriales locales en même temps qu'elles peuvent les freiner, voire les geler, ce qui empêche de considérer tout à fait la structure comme un adjuvant de la construction territoriale. On pense aux nombreuses associations de « défense » qui se créent en Aveyron, en Provence et partout en France. L'Association de Défense des Villages du Moyen Var, basée à Entrevaux (Alpes-de-Haute-Provence), constitue, par exemple, un outil intéressant de protection d'une vallée et de réflexion quant à son devenir environnemental et économique. Dans le même temps, par ses prises de positions tranchées et les conflits interpersonnels qu'elle a parfois envenimés, elle s'est avérée être un obstacle à la mise en place d'un débat serein et équilibré au plan local.

L’interpénétration ou la juxtaposition d'actions associatives sur un même territoire peut aussi aller à l'encontre de son développement : cette superposition peut créer des conflits et bloquer les dynamiques territoriales, au moins momentanément. À Reillanne, de nombreuses associations issues des mêmes domaines d'intervention coexistent, notamment dans le secteur culture-patrimoine-environnement. Composé de structures petites comme Alauza (Sainte-Croix-à-Lauze), moyennes comme Patrimoine de Vachère (Vachères) ou importantes comme Alpes de Lumière (Mane), le tissu associatif s'est ainsi parfois trouvé empêtré dans des rivalités sans issues. Les structures locales n’ont pas toujours été alliées, elles se sont parfois déchirées, s'enfermant dans leurs préoccupations respectives. Or, cela a retardé le développement de quelques chantiers.

Certaines structures souffrent parfois du manque de lisibilité de leur action. Cela ne signifie pas que les associations en question voilent une partie de leurs activités ou cachent un objectif inavoué. Simplement, leurs actions sont quelques fois si hétéroclites qu’elles perdent en cohérence et en intelligibilité. Elles deviennent des sortes d'associations à tout faire, instruments d'action de leur seul responsable. Bien qu'axée sur l'environnement et le patrimoine, l'association SudAveyronnaise des Ateliers du Tayrac (Aveyron) développe des actions liées à la culture à travers l'édition, l'organisation de manifestations musicales, etc. Cela multiplie ses centres d'intérêts et augmente sa visibilité mais réduit aussi son impact, du fait même de sa dispersion.

Enfin, l'instrumentalisation des associations par les élus ou notables locaux, mais aussi la lourdeur et les lacunes de la machine administrative locale, réduisent parfois la marge de manœuvre associative. Prenons l'exemple de la mise en place des Pays institutionnels. Les associations ont leur place dans le projet et intègrent, sur le papier comme sur le terrain, le Conseil de Développement. Pourtant, la question se pose de savoir si leur intégration et surtout leur participation vont demeurer effectives au fur et à mesure de l'avancement du projet. Au stade actuel de la définition des Pays de Haute-Provence (en Luberon), Pays Sisteronnais-Buëch et Pays des Trois V (VerdonVar-Vaïre, dans la vallée du Var), les associations restent mobilisées, mais cela va-t-il durer? Jusqu’à quel point leurs avis et leurs positionnements seront-ils respectés? G. Billard (2003) s'est intéressé à la démarche de Pays. Dans la mesure où son travail s'appuie sur un exemple localisé, en HauteNormandie, il n'est pas représentatif de l'ensemble des situations françaises. Pour autant, il a le mérite de soulever la question de l'implication réelle des associations dans ces territoires de projet et avance quelques limites intéressantes puisqu'elles font écho à des propos relevés en Provence. Les Pays sont-ils des lieux de reproduction des démarches antérieures d'aménagement, intégrant peu les logiques participatives ou sont-ils, au moins pour certains, des lieux où l'on peut observer l'émergence de pratiques nouvelles? Aucun doute, selon lui, les jeux de pouvoir, notamment une crainte constatée des élus politiques locaux de perdre une part de leur légitimité, cadenassent les possibilités de réaliser pleinement les étapes de la participation. Cela rejoint les propos du président de l'Association de Défense des Villages du Moyen Var, à Entrevaux, pour qui, non seulement le Conseil de Développement « ne fait que reproduire en réduction le contexte politique et les conflits locaux », mais en plus donne « la part belle aux élus », qui auraient « le pouvoir de s’imposer dans le Conseil en écartant les structures opposantes...». 


\section{Conclusion}

Le pouvoir des associations en matière de construction territoriale n'est donc pas infini. Ces structures peuvent rencontrer de nombreux obstacles dans leur mobilisation, qui font d'elles des acteurs territoriaux souvent incompris, oubliés (Gumuchian et al., 2003), quelques fois désordonnés, voire bâillonnés... Cela ne signifie pas qu'elles soient dans l'incapacité totale de mener à bien les actions entreprises. Simplement, elles achoppent parfois sur des résistances locales, externes ou mêmes internes, s'effaçant devant des entreprises plus à même de mobiliser leurs moyens pour la réalisation de projet mais moins souples et certainement intéressées ou des établissement publics, parfois mieux dotés qu'elles, mais souvent à la merci des pouvoirs en place... Plus que leur faculté à porter un projet, c'est alors le rôle incontestable des associations comme mobilisateurs au plan local que l'on retient. Car si les associations ne vont pas toujours au bout de leur démarche, elles savent, on l'a vu, faire converger les enthousiasmes jusqu'à amorcer des processus socio-culturels, économiques et politiques, matériels ou idéels, porteurs pour la ruralité et moteurs pour les ruralités, qui feront des territoires ruraux de demain des territoires vivants.

\section{Bibliographie}

Agulhon (M.), Bodiguel (M.), 1981. - Les associations an village, Arles, Actes Sud, coll. "Bibliothèque des Ruralistes », p. 9-38.

Archambault (E.), 2001. - « La loi 1901 sur les associations. L'originalité du modèle français », Problèmes économiques, $\mathrm{n}^{\circ} 2712$, p. 15-18.

Bachelard (P.) (dir.), 1993. - Les acteurs du développement local, Paris, L'Harmattan, coll. Alternances et Développements, $190 \mathrm{p}$.

Billard (G.), 2003. - « La place des associations dans le développement local : l'exemple de la démarche pays en Haute-Normandie ", dans Territoires institutionnels, territoires fonctionnels, actes du colloque de Mâcon, 25-26 sept. 2003, 6 p.

Cabanes (R.), 1986. - «Les associations créatrices de la localité », dans L'Esprit des Lieux. Localités et changement social en France, Paris, Éditions du CNRS, p. 209-231.

Chosson (J.-F.), 1990. - Les générations du développement rural, 1945-1990, Paris, Librairie générale de droit et de jurisprudence, coll. Décentralisation et développement local, 291 p.

Clairet (S.), 2000. - « La Provence, quelle image pour quel territoire? », La Pensée du Midi, n 1, p. 104107.

Conseil national de la vie associative, 2003. - Bilan de la vie associative 2000-2002, Paris, La Documentation Française, 299 p.

Crenner (E.), 1997. - « Le milieu associatif de 1983 à 1996. Plus tourné vers l'intérêt individuel », INSEE Première, $n^{\circ}$ 542, 4 p.

Di Méo (G.), 1991. - L’Homme, la société, l'espace, Paris, Economica, coll. « Géographie », 319 p.

Épagneul (M.-F.), Mathieu (N.), 2000. - « Explorer le rôle des associations dans l'insertion et la création d'emplois en milieu rural », Économie rurale, n 259 , p. 16-32.

FAKI (C.), 1997. - Agriculture et activités nouvelles : facteurs de dynamisme du monde rural, Conseil Économique et Social, direction des Journaux Officiels, 238 p.

Favey (E.), Pellerin (S.), 1999. - « Des territoires en mouvement », Territoires, nº hors-série, p. 4-6.

Fougerouse (C.), 1996. - Le renouveau rural. Dépendance ou autonomie, Paris, L’Harmattan, 378 p.

Gumuchian (H.), 2000. - « Vers la fin de l'espace rural ? L'affirmation des territoires ruraux », Géocarrefour, $n^{\circ} 75-1$, p. 11-12.

Gumuchian (H.), Grasset (E.), Lajarge (R.), Roux (E.), 2003. - Les acteurs, ces oubliés du territoire, Paris, Anthropos, coll. «Géographie », 186 p. 
Nyssens (M.), 1998. - « Le développement des services de proximité : vers une économie plurielle? le cas de la Belgique », dans Nyssens (M.) (dir.), La socio-économie des territoires, Expériences et théories, Paris, L'Harmattan, p. 177-203.

Perrier-Cornet (P.) (dir.), 2002. - Repenser les campagnes, La Tour-d'Aigues, L'Aube/Datar, coll. « Monde en cours », $280 \mathrm{p}$.

Regourd (E.), 2004. - Le phénomène associatif dans la recomposition rurale : vers de nouvelle ruralité?, Thèse de doctorat de géographie, Université Paul-Valéry Montpellier III, 623 p.

Rémy (J.), 1999. - «Dédoublement des espaces sociaux et problématiques de l'habitat », dans BonNiN (P.), Villanova (R. de) (dir.), D'une maison l'autre. Parcours et mobilités résidentielles, Paris, Créaphis, p. 315-346.

SANSELme (F.), 2001. - «Entre sciences sociales et sens commun. La "ruralité" dans les Maisons familiales rurales », Économie rurale, n 262 , p. 62-75.

Sensebe (Y.), 2002. - « Les manifestations contrastées de l'appartenance locale », dans SyLvestre (J.-P.) (dir.), Agriculteurs, ruraux et citadins. Les mutations des campagnes françaises, Dijon, CRDP de Bourgogne/Éducagri, p. 293-310.

Verriele (P.), Martin (J.-F.), 1994. - «Les associations sont-elles en danger? », L’Acteur rural, n 7 , p. 31-46.

Cet article a été reçu le 20 novembre 2006 et définitivement accepté le 20 juin 2007. 
TEXTE HORS DOSSIER THÉMATIQUE 
